\title{
AVALIAÇÃO CLÍNICA DO QUININO PARA OTRATAMENTO DE MALÁRIA POR PLASMODIUM FALCIPARUM
}

\author{
Marcos Boulos, Araripe Pacheco Dutra, Silvia Maria DiSanti, \\ Mário Shiroma e Vicente Amato Neto
}

\begin{abstract}
O quinino foi o primeiro medicamento correntemente usado para tratar malária, tendo sido abandonado seu emprego principalmente após o início do emprego da cloroquina. A partir da década de 60 com o surgimento de resistência do P. falciparum à cloroquina voltou-se a utilizar o quinino isolado ou em associação para tratar tal infecção. Com o objetivo de avaliar clinicamente a resposta ao quinino de pacientes com malária por $\mathrm{P}$. falciparum, analisamos os prontuários de 484 pacientes atendidos no Laboratório de Malária da SUCEN e acompanhados por pelo menos 28 dias, e que haviam recebido diferentes esquemas terapêuticos com quinino isolado ou em associação. Do total, 81,0\% dos pacientes foram curados pelos esquemas empregados, sendo que dos restantes apenas $0,6 \%$ foram $R 2$ e nenhum $R 3$. Tais resultados mostram ainda que esquemas contendo quinino podem ser adequados para tratar malária por P. falciparum.

Palavras-chaves: Malária. Tratamento. Plasmodium falciparum. Quinino. Resistência.
\end{abstract}

A malária causada por $P$. falciparum é aquela em que o médico encontra maiores dificuldades no seu tratamento devido ao rápido surgimento de resistência aos diferentes esquemas empregados.

Assim é que foi surgindo resistência do $P$. falciparum sucessivamente ao quinino, cloroquina, associação da sulfadoxina e pirimetamina e mais recentemente à mefloquina e derivados da artemisinina.

Quando do surgimento de resistência aos esquemas habitualmente empregados pelo Ministério da Saúde nas décadas de 60 e 70 (cloroquina, sulfadoxina e pirimetamina), o quinino, cujo emprego havia sido abandonado, voltou a ser utilizado isolado ou em associação.

A utilização do quinino havia sido interrompida não só devido ao surgimento de resistência, que não era muito elevada, mas

\footnotetext{
Departamento de Doenças Infecciosas e Parasitárias da Faculdade de Medicina da Universidade de São Paulo. SUCEN - Superintendência de Controle de Endemias da Secretaria de Estado da Sa de de São Paulo.

Endereço para correspondência: Dr. Marcos Boulos. Av. Enéias Carvalho de Aguiar 470, sala 33, 05403-000 São Paulo, SP. Fax: (011) 881-8158.

Recebido para publicação em 24/07/96.
}

também devido a efeitos tóxicos do medicamento e a baixa operacionalidade do mesmo (ministrado em pelo menos 7 dias)

Com o objetivo de conhecer a resposta de pacientes com malária por P. falciparum tratados com associações de medicamentos contendo quinino, foi realizado o presente estudo.

\section{MATERIAL E MÉTODOS}

Foram analisados os prontuários de 484 pacientes adultos com malária por $P$. falciparum atendidos no Laboratório de Malária da SUCEN, no período de janeiro de 1982 a dezembro de 1988 , tratados com esquemas contendo quinino, acompanhados por pelo menos 28 dias.

Todos os prontuários analisados referem-se a pacientes com baixa parasitemia (abaixo de 2\% das hemácias), sem complicações, e que puderam ser acompanhados ambulatorialmente.

Os esquemas utilizados para esses pacientes foram: sulfato de quinino isoladamente durante 7 dias na dose de 500mg a cada 8 horas, em 126 pacientes, sulfato de quinino na dose de 500mg a cada 8 horas durante 3 dias associado à sulfadoxina na dose única de $1,5 \mathrm{~g}$ e pirimetamina na dose única de $50 \mathrm{mg}$, em 119 pacientes e sulfato de quinino na dose de $500 \mathrm{mg}$ a cada 8 horas durante 3 dias associado 
Boulos M, Dutra AP, DiSanti SM, Shiroma M, Amato Neto V. Avaliação clínica do quinino para o tratamento de malária por Plasmodium falciparum. Revista da Sociedade Brasileira de Medicina Tropical 30:211-213, mai-jun, 1997.

à tetraciclina na dose de $500 \mathrm{mg}$ a cada 8 horas durante 7 dias, em 239 pacientes.

Para a interpretação da resposta clínica empregou-se a mesma classificação utilizada para estudos com a cloroquina e sugerida pela Organização Mundial da Saúde, ou seja, sensivel (S) quando o paciente foi curado pelo esquema proposto, acompanhado por 28 dias, R1 quando após desaparecimento da parasitemia assexuada no sangue periférico ocorreu reaparecimento da mesma até 28 dias do tratamento, R2 quando houve significativa diminuição da parasitemia assexuada durante a primeira semana de tratamento, sem seu desaparecimento, e R3 quando houve pequena diminuição da parasitemia assexuada ao exame periférico ou não houve diminuição da mesma no período de 7 dias.

A SUCEN atende pacientes com malária procedentes de diferentes regiões do país e os resultados que foram obtidos podem ser considerados representativos das áreas mais malarígenas do Brasil (Rondônia, Mato Grosso e Pará).

\section{RESULTADOS}

O resultado obtido pelo acompanhamento dos pacientes encontra-se esquematizado na Tabela 1.

Tabela 1 - Resposta clínica ao tratamento com esquemas com quinino em pacientes com malária por Plasmodium falciparum.

\begin{tabular}{|c|c|c|c|c|c|c|c|c|c|}
\hline \multirow[t]{2}{*}{ Esquemas } & \multicolumn{2}{|c|}{ S } & \multicolumn{2}{|c|}{ R1 } & \multicolumn{2}{|c|}{ R2 } & \multicolumn{2}{|c|}{ R3 } & \multirow[t]{2}{*}{ Total } \\
\hline & $\mathrm{n}^{\mathrm{O}}$ & $\%$ & $\mathrm{n}^{\mathrm{O}}$ & $\%$ & $\mathrm{n}^{\mathrm{O}}$ & $\%$ & $\mathrm{n}^{\mathrm{O}}$ & $\%$ & \\
\hline $\bar{Q}$ & 97 & 77,0 & 28 & 22,3 & 1 & 0,8 & - & - & 126 \\
\hline QSP & 74 & 62,2 & 43 & 36,1 & 2 & 1,7 & - & - & 119 \\
\hline QT & 221 & 92,5 & 18 & 7,5 & - & - & - & - & 239 \\
\hline Total & 392 & 81,0 & 89 & 18,4 & 3 & 0,6 & - & - & 484 \\
\hline
\end{tabular}

Como se pode ver houve diferença de resposta quando foi utilizado quinino isoladamente ( $77 \%$ de cura e $0,8 \%$ de R2), quinino associado à sulfadoxina e pirimetamina $(62,2 \%$ de cura $\mathrm{e}$ $1,7 \%$ de R2) e quinino associado à tetraciclina (92,5\% de cura, sem R2).

\section{DISCUSSÃO}

O quinino voltou a ser utilizado no Brasil com a disseminação de resistência do P. falciparum à cloroquina e à associação de sulfadoxina e pirimetamina, a partir de meados dos anos 802 .

A despeito do quinino ter sido empregado isoladamente em várias ocasiões, foi sugerido que houvesse a utilização dessa droga associada a outros medicamentos com intuito de reduzir a conhecida resistência a ele, como também de diminuir a número de doses do quinino com o objetivo de reduzir a toxicidade.

Modelo de associação de quinino com a tetraciclina inicialmente empregado na Tailândia também foi utilizado no Brasil com sucesso 24. Tal combinação procura associar esquizonticida sangüíneo rápido (quinino) com outro lento (tetraciclina).

Aparentemente, a pior resposta encontrada com a associação de quinino com sulfadoxina e pirimetamina, quando comparada ao quinino, se deve ao fato de se utilizar quinino por apenas 3 dias nessa associação e corrobora com a conhecida resistência disseminada do $P$. falciparum à associação sulfadoxina-pirimetamina3.

A pior resposta ao quinino, quando comparada à associação quinino-tetraciclina, se deve à gradativa lentificação de resposta daquela droga com o transcorrer dos anos como foi observado no Brasil1 e na Tailândia45.

A associação de quinino e tetraciclina que nestes pacientes mostrou ser bastante eficaz, foi utilizada em vários locais para tratar malária falcípara resistente também com bons resultados 2345 .

Um óbice do emprego das associações com quinino decorre da pequena operacionalidade do emprego de tais esquemas, já que se faz necessário utilização prolongada de antimaláricos, dificultando seu uso.

Os dados aqui apresentados, reiteram o valor do quinino no tratamento de malária por $P$. falciparum não grave, quando não existe grande premência na negativação da parasitemia.

\section{SUMMARY}

Quinine was the first antimalarial drug to be employed and also the first resistance was noticed to. After 1960' quinine urged to be reintroduced in routine therapy alone or in combination. Aiming at evaluating the effectiveness of different schedules we studied 484 patients seen at the Malaria Laboratory. We used quinine alone in 126 patients, quinine plus sulfadoxine and pyrimethamine in 119 patients and quinine plus tetracycline in 239 patients. The results shown that $81 \%$ of all patients were treated with success and only $0.6 \%$ were R2, and there is no $R 3$. We emphasize a high resistance rate to quinine either alone (23.1\%) or associated to sulfadoxine and pyrimethamine (37.8\%). A bigher resistance 
Boulos M, Dutra AP, DiSanti SM, Shiroma M, Amato Neto V. Avaliação clínica do quinino para o tratamento de malária por Plasmodium falciparum. Revista da Sociedade Brasileira de Medicina Tropical 30:211-213, mai-jun, 1997.

rate seen with the combination might be linked to the smaller dose of quinine used in that instance. It is worth noting the high cure rate with the quininetetracycline association.

Key-words: Malaria. Treatment. Plasmodium falciparum. Quinine. Resistance.

\section{REFERÊNCIAS BIBLIOGRÁFICAS}

1. Albuquerque BC, Alecrim WD, Dourado HV, Penha AMM, Silva VMFQ, Sampaio LS, Sampaio MZM. Resposta a sensibilidade do P. falciparum in vivo ao quinino na Amazonia Brasileira. In: Resumos do XXI Congresso da Sociedade Brasileira de Medicina Tropical, São Paulo p. 130, 1985.

2. Barata LCB, Boulos M, Dutra AP. Emprego da associação tetraciclina e quinino no tratamento da malária causada pelo Plasmodium falciparum. Revista da Sociedade Brasileira de Medicina Tropical 19:135-137, 1986.
3. Boulos M, DiSanti SM, Barata LCB, Segurado AAC, Dutra AP, Camargo-Neves VLF. Some aspects of treatment, prophylaxis and chemoresistance of Plasmodium falciparum malaria. Memórias do Instituto Oswaldo Cruz 81(supl II):255-257, 1986.

4. Pinichpongse S, Doberstyn EB, Cullen JR, Yisunsri L, Thongsombun Y,Thimasarn K. An evaluation of five regimens for the outpatient therapy of falciparum malaria in Thailand 1980-1981. Bulletin of the Worls Health Organization 60:907912, 1982.

5. Suebsaeng L, Wernsdorfer WH, Rooney W. Sensitivity to quinine and mefloquine of Plasmodium falciparum in Thailand. Bulletin of the World Health Organization 64:759-765, 1986. 